\title{
Effects of Innovative Suction Slots on the Performance of a Radial Bladed Impeller of a Centrifugal Blower - A Numerical Transient Analysis
}

\author{
Madhwesh N and K Vasudeva Karanth*
}

\begin{abstract}
The recirculation zones present in between the blade passages give rise to some losses in centrifugal blowers. The main objective of this study is to realign the flow at the suction side of the impeller by weakening the recirculation zones. This can be achieved by providing slots on the impeller blade geometry, which will efflux the flow from the pressure side of the impeller towards its suction side. Effects of single and multiple slots were analyzed numerically for possible pressure recovery. The comparison of performance among various slot configurations is represented in terms of non-dimensional parameters. It is found from the numerical study that a single slot provided at a radial distance of $75 \%$ from the blade root and twin slots located at $50 \%$ and $75 \%$ from the leading edge of the impeller vane significantly contribute to the static pressure recovery across the fan. Hence, it is beneficial to use multiple suction slots for better performance augmentation.
\end{abstract}

Keywords: Centrifugal blower; Flow separation; Multiple slots; Slot size; Transient analysis.

Received: 21 September 2021; Revised: 03 December 2021; Accepted: 04 December 2021.

Article type: Research article.

\section{Introduction}

The suction slots are holes in the geometry of the impeller blades, channeling enough fluid from the pressure side toward the suction side. They are positioned at the point of separation to control the boundary later build-up. Fluid effluxes through the slot result in moving the separation region closer to the tip of the impeller, thus reducing the slip as well as flow losses. This method is very effective for reducing leading edge suction peaks in the partial capacity operation range. Hence, in the present work, an effort is made to perform the numerical analysis on the slotted impeller, which drives toward a possibility of overall performance augmentation in terms of static pressure recovery across the blower.

$\mathrm{K}$. Hubrich et al. ${ }^{[1]}$ found that the working range of the transonic compressor could be increased by leaching a small amount of flow through suction slots and the maximum pressure ratio and diffusion could both be increased satisfactorily. Shojaefard et al. ${ }^{[2]}$ studied flow field over a subsonic airfoil with some suction and injection slots, on the

\footnotetext{
Department of Mechanical and Manufacturing Engineering, Manipal Institute of Technology, Manipal Academy of Higher Education, Manipal 576104, Karnataka, India.

*Email: kv.karanth@manipal.edu (K. V. Karanth)
}

vacuum side of the airfoil. They found that the surface suction increased the lift coefficient significantly while the injection slot decreased the skin friction. Smith et al. ${ }^{\left[{ }^{[3]}\right.}$ theoretically examined 2D/3D surface suction slots inside a planer boundary layer. They found that on increasing the suction strength, non-linear structures were enhanced with flow reversals or trailing vortices. Song et al..$^{[4]}$ studied the influence of boundary layer suction on the performance of compound lean compressor cascades. They found that the total loss of cascades was reduced significantly by boundary layer suction. They concluded that the slot position along the full span proved to be the best. Georgiev et al..$^{[5]}$ studied the flow field differences by perforation and slotting of the impeller blade. They found that these modifications reduced power requirements and increased deformation impacts. Karanth et $a l^{[6]}$ studied the effect of boundary layer suction slots at various geometrical locations on the impeller. They found that the suction slots provided on the impeller blade near its trailing edge significantly improved the static pressure recovery across the fan. They also conducted a study with converging slots. ${ }^{[7]}$ They found that the converging suction slots located on the impeller blade near the trailing edge appreciably improved the static pressure recovery across the fan.

Khalid $^{[8]}$ conducted an experimental study by using slots in 
the impeller blades at some shroud distance from the exit of the blade. He found that by minimizing the boundary layer growth near the exit of the blade, the stable margin was increased. He also found that the deriving power of the compressor was decreased due to a reduction in the impeller losses. Madhwesh et al. ${ }^{[9]}$ examined the effects of splitter vanes at various geometrical locations on the impeller blade. They found that the splitter vanes located near the leading edge improved the static pressure recovery of the fan. They also commented that the splitter vanes provided near the trailing edge of the impeller adversely affected the static pressure recovery of the fan. Dovgal et al. ${ }^{[10]}$ conducted a wind tunnel study of the influence of flow suction on a laminar boundary layer separation. They found that this method of flow control suppresses the formation of large-scale vortices determined by the global stability properties of the separation region.

Gancedo $\mathrm{M}$ et al. ${ }^{[11]}$ described the effect of bleed slots on the enhancement of surge margin in a centrifugal compressor of a turbocharger. They dealt with the study of flow instabilities and the consequent effect of unstable flow on compressor maps, especially at lower mass flow rates. It is found that by providing the bleed slot stability of the compressor gets improved. Ren-hui Zhang et al. ${ }^{[12]}$ performed a Computational Fluid Dynamics (CFD) analysis on a centrifugal pump. The primary focus was to minimize the tendency of cavitation by providing a slot on the impeller shroud near the suction side of the leading edge of the blade. Their research showed that the slot jet plays a vital aspect in diminishing cavitation and flow reversal.

Hongliang Wang et al. ${ }^{[13]}$ conducted a numerical analysis on a centrifugal pump having a slotted impeller. Various geometries of the slot in terms of slot size, slot position, and slot deflection angle were analyzed numerically. They found that slot position and deflection angle plays a major role in influencing the efficiency of the pump.

With a thorough careful literature review, it is found that there has not been much focus given to multiple suction slots in the impeller of the centrifugal turbomachine. This research gap is identified and implemented in the present numerical analysis. The jet-like flows emerging out of the multiple slots are expected to trigger the weakening of the recirculation zones in the impeller passages, thereby enhancing the overall performance of the device.

\section{Methodology}

\subsection{Geometric specifications}

Figure 1 shows the schematic diagram of the centrifugal blower used to conduct numerical analysis. The flow domain consists of three regions: Inlet, impeller, and volute. Specification of the blower is depicted in Table 1.

The schematic numerical model is built with the help of ANSYS, and the domains used for analysis are shown in Fig. 2.

\subsection{Slot geometry}

A judicial position of the slots must be decided such that a portion of fluid efflux from the slots delays boundary layer separation. Various configurations of slots used in the present analysis are depicted in Fig. 3. The configurations M1, M2, and M3 represent single slot arrangements on the impeller blades whereas M4, M5, and M6 signify twin slot arrangements. The radial height of the suction slot is varied from $2 \mathrm{~mm}$ to $6 \mathrm{~mm}$ in the step of $1 \mathrm{~mm}$ for all the configurations used.

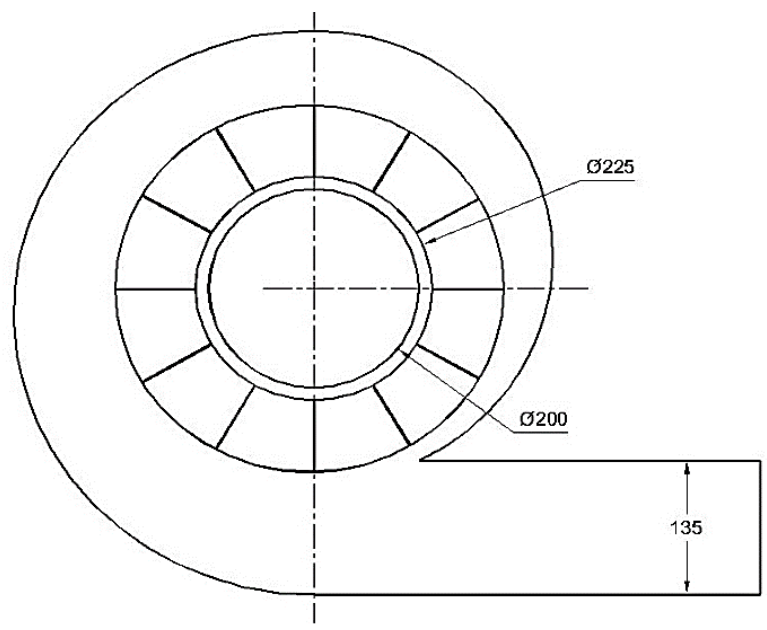

Fig. 1 Schematic diagram of the centrifugal blower.

Table 1. Specification of the centrifugal blower.

\begin{tabular}{ll} 
Inlet diameter of the blower & $200 \mathrm{~mm}$ \\
Inlet diameter of the impeller & $225 \mathrm{~mm}$ \\
The outer diameter of the impeller & $370 \mathrm{~mm}$ \\
Vertical channel height of volute & $135 \mathrm{~mm}$ \\
Number of impeller blades & 12 \\
The thickness of the impeller blade & $2.5 \mathrm{~mm}$ \\
\hline
\end{tabular}

(a)

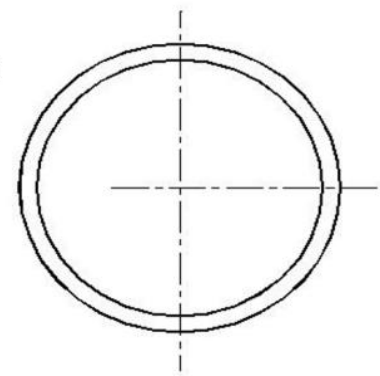

(b)

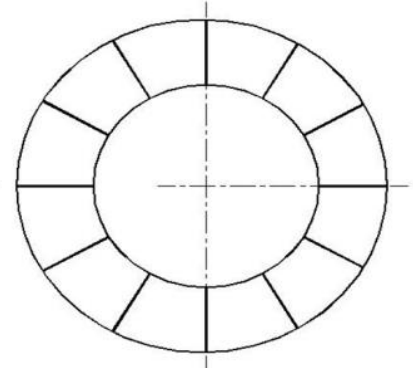

(c)

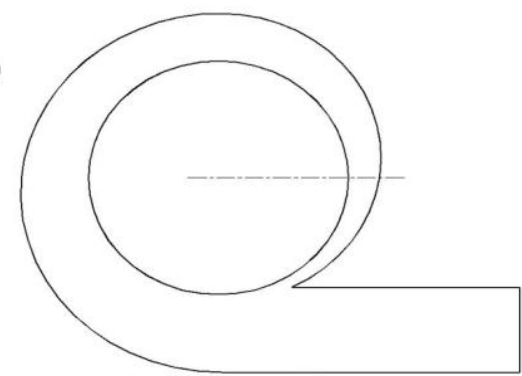

Fig. 2 (a) Inlet region, (b) Impeller passage and (c) Volute casing. 
(a)

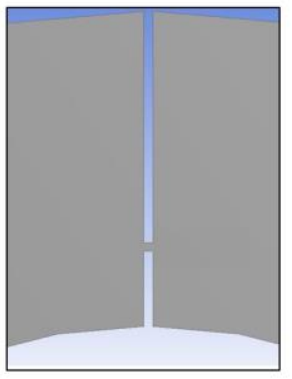

(d)

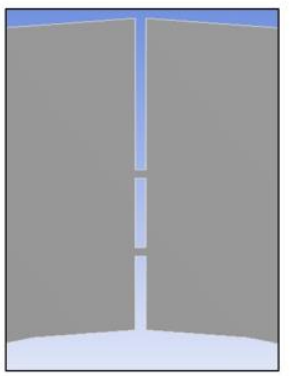

(b)

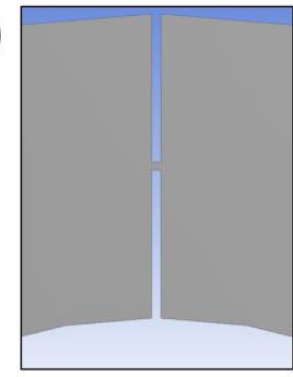

(e)

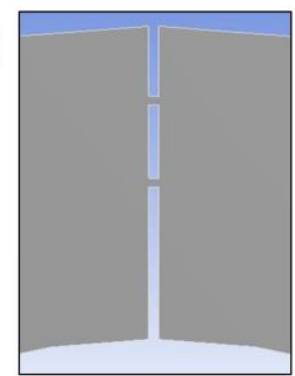

(c)

(f)

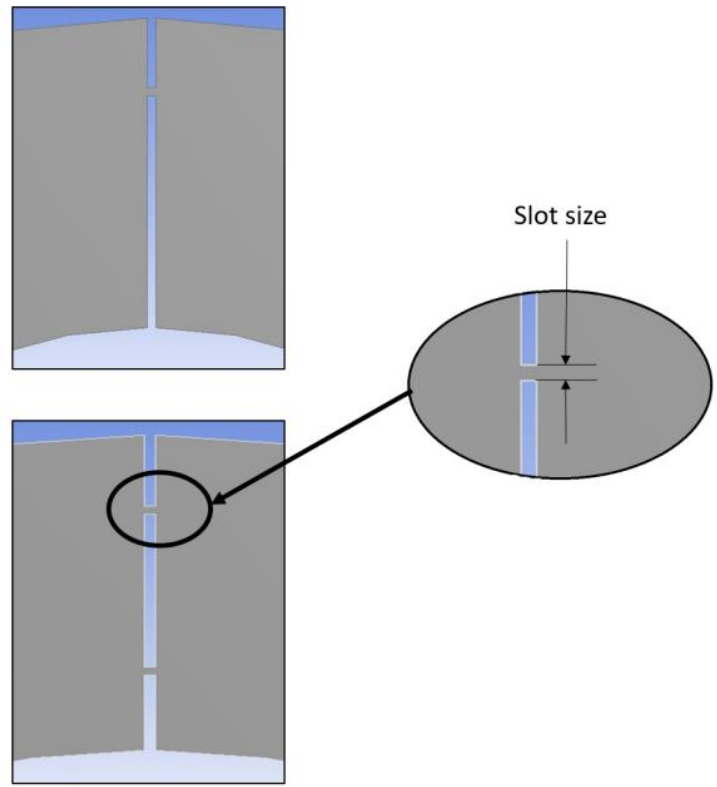

Fig. 3 Slot configurations used in the present study: (a) Configuration M1, (b) Configuration M2, (c) Configuration M3, (d) Configuration M4, (e) Configuration M5, and (f) Configuration M6.

Configurations M1 depicts the presence of a slot at a meridional height of $25 \%$ from the inlet of the impeller whereas M2 has a slot at the mid-height of the impeller. The slot is located at $75 \%$ of the radial height from the impeller root for configuration M3. Configurations M4 represents the presence of slots at $25 \%$ and $50 \%$ of the radial height from the inlet of the impeller. The slots are provided at the mid-height of the impeller and $75 \%$ of the blade height for configuration M5. Slots are located at $25 \%$ as well as $75 \%$ of the radial height from the impeller root for configuration M6.

\subsection{Boundary conditions and unsteady analysis}

An inlet velocity of $5 \mathrm{~m} / \mathrm{s}$ is imposed for the inlet as per the previous work done by Karanth et al. ${ }^{\left[{ }^{[6]}\right.}$ The turbulence intensity is set to a lower value of $5 \%$. The flow in this blower is inherently turbulent as the Reynolds number is around 64000 corresponding to the given inlet velocity. The turbulence model used is $\mathrm{K}$ - the epsilon model with standard wall treatment which is a widely accepted and computationally reliable model used in CFD analysis for turbomachinery applications..$^{[6,14]}$ An outflow boundary condition is set assuming that the flow is fully developed at the exit of the blower. The no-slip boundary is specified for blades and walls of the volute as a prescribed boundary condition. Second order upwind scheme is used for spatial discretization for momentum, turbulence kinetic energy, and turbulence dissipation rate. Pressure velocity coupling is done using a simple algorithm. The solver is pressure based, as the flow is in a subsonic regime.

For the transient simulation, a sliding mesh method is used in which mesh interfaces are created between the inlet out and impeller inlet, impeller outlet, and volute inlet. The sliding mesh works by updating the relative position of the impeller with respect to the volute and inlet. This updating in position will help in capturing rotor and stator interactions. The time step is $6.67 \mathrm{e}-5 \mathrm{~s}$, which corresponds to one degree of impeller rotation at the rated speed of 2500 RPM to establish stability criteria. The maximum iterations per time step are/given as 20 so that the largest residuals reduce below 1e-5. As the flow is unsteady, it is desirable to carry out iterations till the important parameters such as static pressure at the outlet, and total pressure at the impeller outlet become time-periodic. For all cases, it is found that the output parameters become time periodic after four complete revolutions of the impeller. The time and area-weighted averages are found for pressure and total pressure at salient locations.

\subsection{Governing equations}

The governing conservative differential equations for continuity, momentum, and scalar transport in the reference frame of the moving mesh may be written in the Cartesian tensorial form and are given in equations (1), (2), (3), and (4) respectively. ${ }^{[14]}$

Continuity equation:

$$
\frac{d}{d t} \rho+\frac{\partial}{\partial x_{j}}\left(u_{j}-v_{j}\right)=0
$$

Momentum equation:

$$
\frac{d}{d t} \rho u_{i}+\frac{\partial}{\partial x_{j}} \rho\left(u_{j}-v_{j}\right) u_{j}=-\frac{\partial p}{\partial x_{j}}+\frac{\partial}{\partial x_{j}}\left(\tau_{i j}+\tau_{t i j}\right)+S_{u i}
$$

The equation for $\mathrm{k}$ :

$$
\frac{\partial}{\partial t}(\rho k)+\frac{\partial}{\partial x_{j}}\left(\rho u_{i} k\right)=\frac{\partial}{\partial x_{j}}\left(\frac{\mu}{\sigma_{k}} \frac{\partial k}{\partial x_{j}}\right)+G_{k}-\rho \varepsilon
$$

Equation for $\varepsilon$ :

$\frac{\partial}{\partial t}(\rho \varepsilon)+\frac{\partial}{\partial x_{j}}\left(\rho u_{i} \varepsilon\right)=\frac{\partial}{\partial x_{j}}\left(\frac{\mu}{\sigma_{\varepsilon}} \frac{\partial \varepsilon}{\partial x_{j}}\right)+C_{1 \varepsilon} \frac{\varepsilon}{k} G_{k}-C_{2 \varepsilon} \rho \frac{\varepsilon^{2}}{k}$ 


\subsection{Generation of the computational model}

A two-dimensional (2D) computational fluid dynamics (CFD) analysis is carried out and is assumed to represent the midspan of the real three-dimensional computational domain. By performing a two-dimensional CFD analysis, it is possible to obtain approximate parameters of the passive control techniques and save considerable computational time. It is assumed that the wall shear effect of the hub and shroud is negligible. With the available geometry specifications 2D computational models of the inlet, impeller, and volute are built by the popular analysis software. These three domains are meshed to capture the flow properties accurately. A grid independence test is conducted to obtain optimum grid size for the purpose of computational time-saving. Static pressure at the exit of the blower is considered a deciding parameter for the grid independence test.

The grid size on the computational domains is varied from $2 \mathrm{~mm}$ to $6 \mathrm{~mm}$ in steps of $1 \mathrm{~mm}$. It is discernable from Fig. 4 that there exists a marginal difference in the value of static pressure between grid sizes of $2 \mathrm{~mm}$ and $3 \mathrm{~mm}$. Hence, a grid size of $3 \mathrm{~mm}$ for all domains of fluid flow is adopted at the mainstream regions of fluid flow in the present research. Finer mesh is given near blade edges which enables to capture of boundary layer effects effectively as these places tend to be the region of the high-velocity gradient. A magnified region of the meshed domain is shown in Fig. 5. The domain-wise statistics of the mesh are depicted in Table 2.

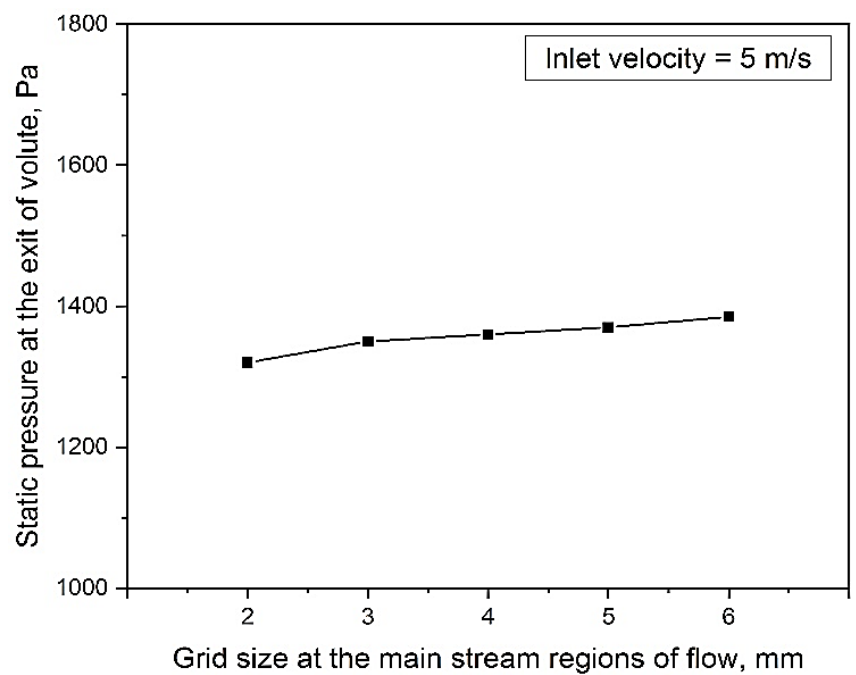

Fig. 4 Grid independence test results.

Table 2. Mesh statistics.

\begin{tabular}{ccc}
\hline Domain & Number of nodes & Number of elements \\
\hline Inlet & 9000 & 7200 \\
Impeller & 81000 & 74760 \\
Volute & 278050 & 271950 \\
Total & 368050 & 353910 \\
\hline
\end{tabular}

\section{Validation of the numerical model}

It is a well-known fact that the CFD model should be validated either through theoretical formulations or well-calibrated experimental work. In the present study numerical work is validated with the help of experimental work conducted on a centrifugal blower.

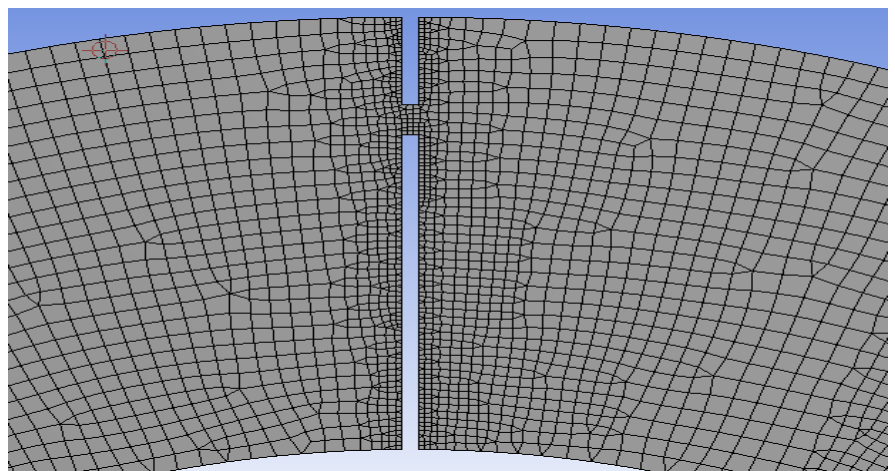

Fig. 5 Magnified regions of the meshed domain.

Experiments are carried out by imposing three arbitrarily chosen absolute velocity values $(3 \mathrm{~m} / \mathrm{s}, 5 \mathrm{~m} / \mathrm{s}$, and $7 \mathrm{~m} / \mathrm{s})$ at the blower inlet. This is achieved by controlling the exit gate valve. The static pressure at the exit of the blower is captured using a well-calibrated pitot tube. The static pressure values from CFD simulations are obtained for the corresponding imposed velocity values. A validation graph for experimental and CFD studies is plotted and is shown in Fig. 6. Table 3 depicts the comparison of CFD and experimental values of static pressure measured at the exit flange of the volute casing, along with the percentage error between them.

It is found from Fig. 6 that, as the velocity increases at the blower inlet, static pressure at the volute also increases experimentally. This means the peak pressure point is not reached yet. Hence, the chosen inlet velocity values are considered to be off-design operation points of the blower.

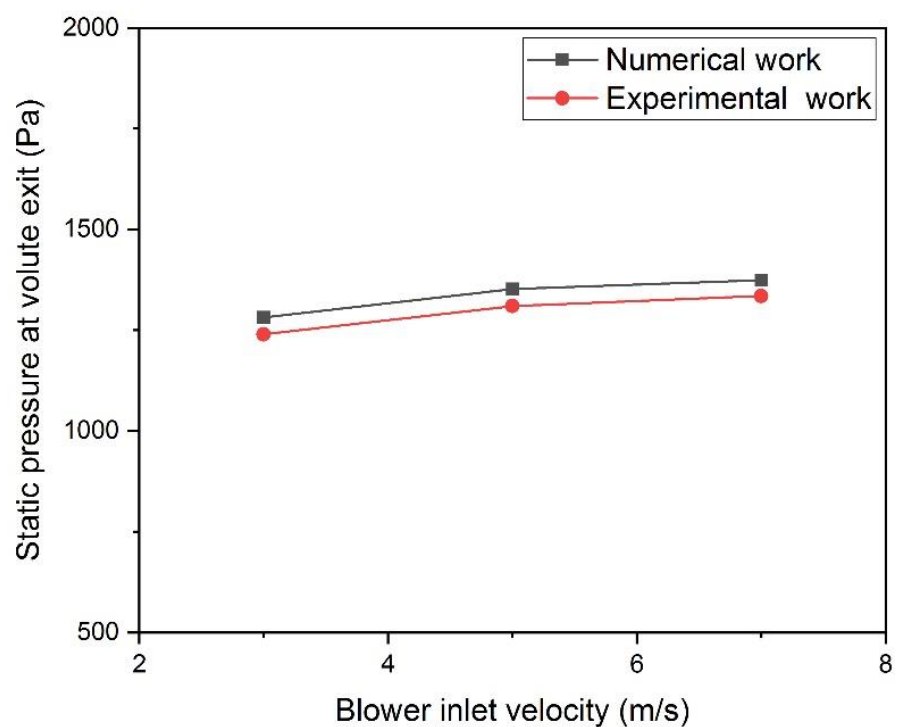

Fig. 6 Validation plot between numerical and experimental work.

Table 3. Percentage error between experimental and CFD results.

\begin{tabular}{llll}
\hline \multirow{2}{*}{$\begin{array}{l}\text { Inlet velocity } \\
(\mathrm{m} / \mathrm{s})\end{array}$} & \multicolumn{2}{c}{ Static pressure at volute exit $(\mathrm{Pa})$} & \multirow{2}{*}{$\begin{array}{l}\text { Percentage } \\
\text { error }\end{array}$} \\
\cline { 2 - 3 } & Experimental value & CFD value & 3.31 \\
3.0 & 1240 & 1281 & 3.04 \\
5.0 & 1314 & 1354 & 3.23 \\
\hline 7.0 & 1332 & 1375 & \\
\hline
\end{tabular}




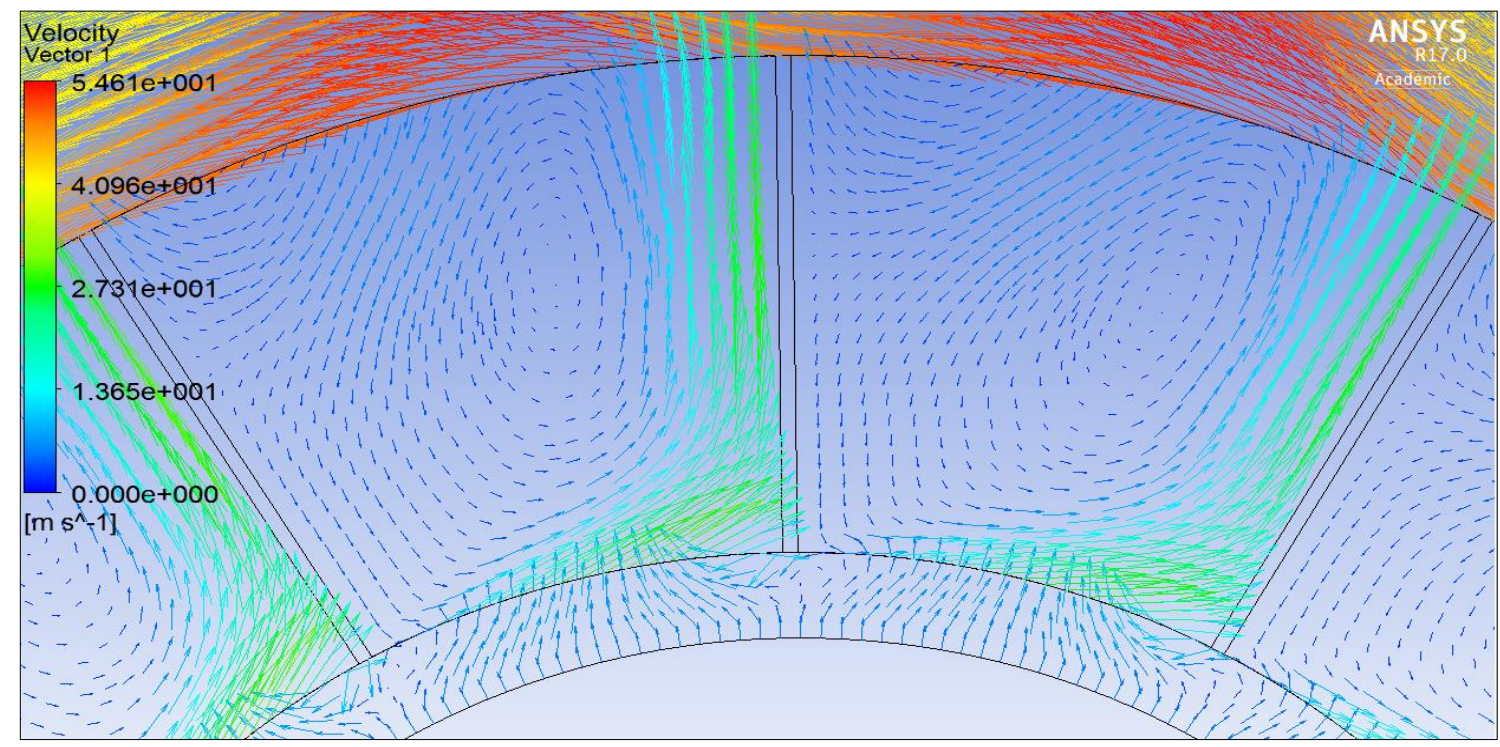

Fig. 7 Velocity vector plot for the base model configuration.

CFD results also follow a similar trend with a marginal variation with respect to experimental values. It is seen in Fig. 6 and Table 3. There exists a minor deviation of about $3.19 \%$ between numerical work and experimental work. Hence it is concluded that CFD results are in conformity with experimental work, with minor deviations due to diffusion effects in the impeller passages, no backflow of air from volute to impeller, and negligence of third-dimensional flow losses. ${ }^{[15]}$

\section{Results and discussion}

The effectiveness of these passive control techniques is evaluated by defining relevant non-dimensional coefficients such as the Overall static pressure rise factor $(\alpha)$ and Overall total pressure loss factor $(\beta)$. These parameters are defined in equations (5) and (6) respectively.

$$
\begin{aligned}
& \alpha=\frac{p_{4}-p_{1}}{\rho u^{2}} \\
& \beta=\frac{p_{t 4}-p_{t 1}}{\rho u^{2}}
\end{aligned}
$$

All the numerical analyses are carried out at a single inlet velocity value of $5 \mathrm{~m} / \mathrm{s}$ as mentioned earlier. The results are discussed in two categories. Firstly, the performance of single slotted impeller is discussed with the help of relevant histograms plotted for performance parameters. Subsequently, the discussion is dealt with performance analysis of twin slot configurations. The quantitative results presented in the histograms are reasoned for each case with the help of useful velocity vector plots captured near the critical regions of interest.

\subsection{Effect of the single slot on the performance}

The velocity vector plot for the base model configuration without a suction slot is shown in Fig. 7. It is seen clearly in Fig. 7 that there exists a strong recirculation zone in the impeller blade passages thereby obstructing the flow of air into the volute casing. This leads to performance losses in terms of static pressure rise.
Figure 8 depicts the variation of the overall static pressure rise factor for various single slot configurations and slot dimensions. It is seen from Fig. 8 that the slotted configurations M2 and M3 seem to perform better in terms of the overall static pressure rise factor when compared with that of the base model. However, configuration M1, corresponding to the slot location in the vicinity of the eye of the impeller, is found to provide an unfavorable effect in terms of pressure recovery of the blower.

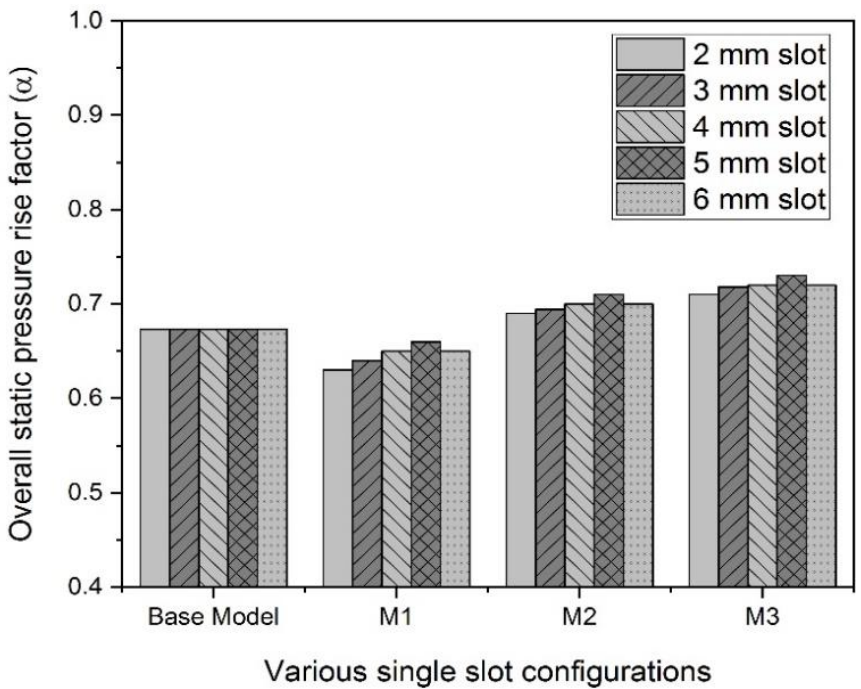

Fig. 8 Plot of overall static pressure rise factor for various single slot configurations and slot sizes.

Among the single-slotted geometric models studied in the analysis, configuration $\mathrm{M} 3$, corresponding to a slot location that corresponds to $75 \%$ of radial distance from the blade root, is found to be the optimum geometric configuration. The reason for the optimum performance is the fact that the slot provided on the impeller blades pushes the fluid on the suction side to improve its momentum thereby causing a through flow in the vicinity of the impeller fluid flow passage. So also, 


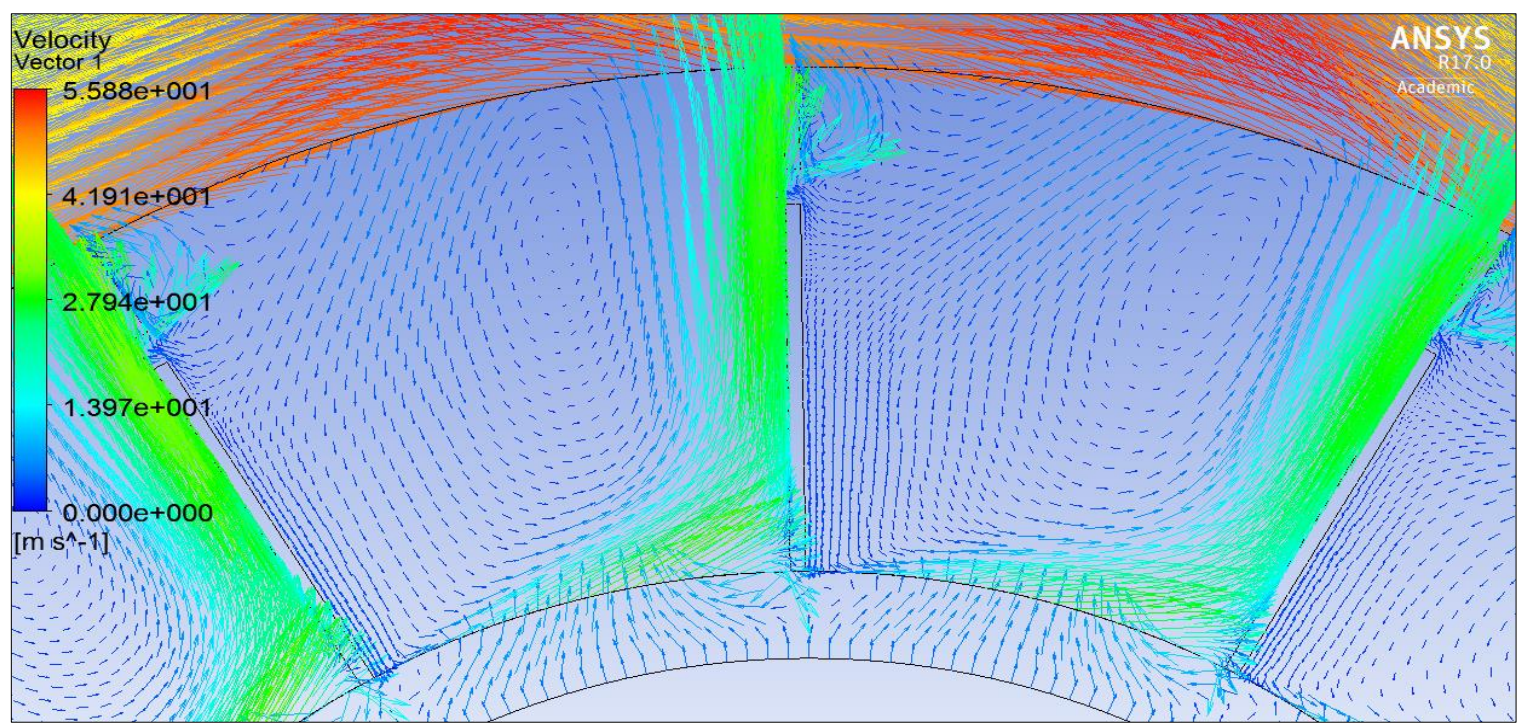

Fig. 9 Velocity vector plot of configuration M3 with optimum slot size.

configuration M3 with a slot radial height of $5 \mathrm{~mm}$ performs better when compared with other slot sizes. This slot size can be considered as optimum slot size in terms of a relatively higher overall static pressure rise factor. This slot width yields about a $5.5 \%$ improvement in the overall static pressure rise factor when compared with the base model configuration. This may be due to the fact that the jet-like flow emerging through this sized slot is very effective in minimizing the detrimental effect of recirculation zones to a certain extent.

The velocity vector plot for the optimum configuration is shown in Fig. 9. It is clearly seen that the jet type of flow partially inhibits the strength of the recirculation zone in the impeller passages, thereby pushing the fluid effectively into volute casing, so that a more streamlined flow is expected in the vicinity of the inlet region of volute casing. This leads to the relatively minimum amount of tip leakage flow losses at the interface thereby contributing towards pressure recovery.
It is seen from Fig. 8 that performance of configuration M2 is marginally better than that of the base model. At the midheight of the blade, the jet effect is balanced and a gentle push is provided by the jet fluid through the slot this results in a slightly higher pressure rise in the impeller as shown in the velocity vector plot for this configuration (Fig. 10).

Contrastingly, it is also seen in Fig. 8, Configuration M1 yields a relatively lower overall static pressure rise factor when compared with the base model configuration for all slot sizes. This can be attributed to the fact that in the vicinity of the impeller eye region, there exist significant disturbances in the flow profile due to the abrupt change in the direction of fluid flow from axial to radial. Further, the slot provided in this region produces additional turbulence for this configuration. This results in the formation of eddies beneath the slot and deteriorates the pressure rise in the impeller as shown in Fig. 11.

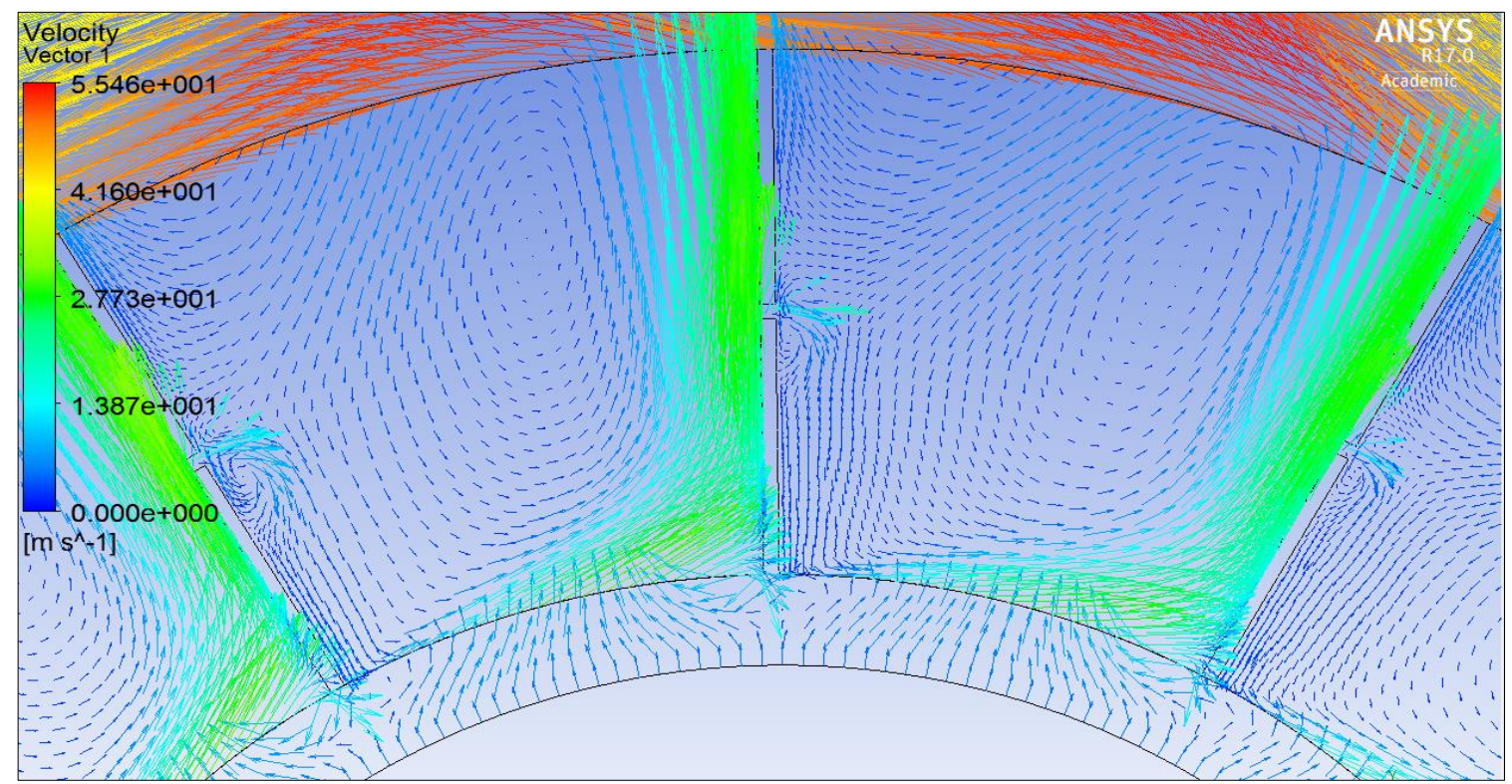

Fig. 10 Velocity vector plot of configuration M2 with optimum slot size. 


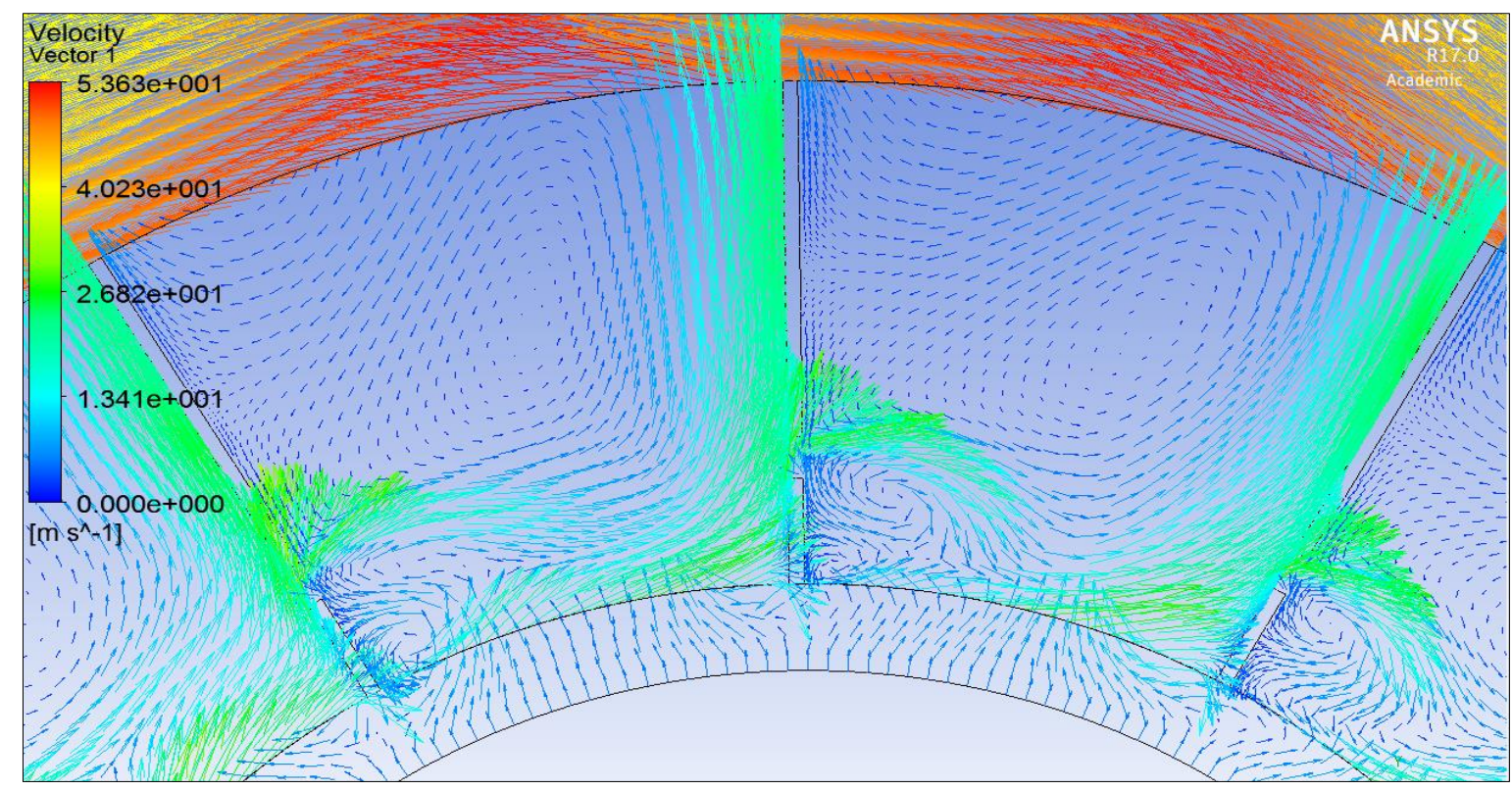

Fig. 11 Velocity vector plot of configuration M1 with optimum slot size.

Additionally, the plot of the total pressure loss factor for all the single-slotted configurations is displayed in Fig. 12. The rise in useful static pressure obtainable from the configurations M2 and M3 is resulting in a relatively lower total pressure loss factor across the blower for these configurations, thereby complimenting and justifying Fig. 8 .

In general, it can be concluded that a single slot provided near the impeller tip improves the performance of the blower by reducing the possible tip clearance losses and streamlining the flow of fluid whereas the slot provided near the eye of the impeller hampers the performance of blower by providing additional recirculation eddies.

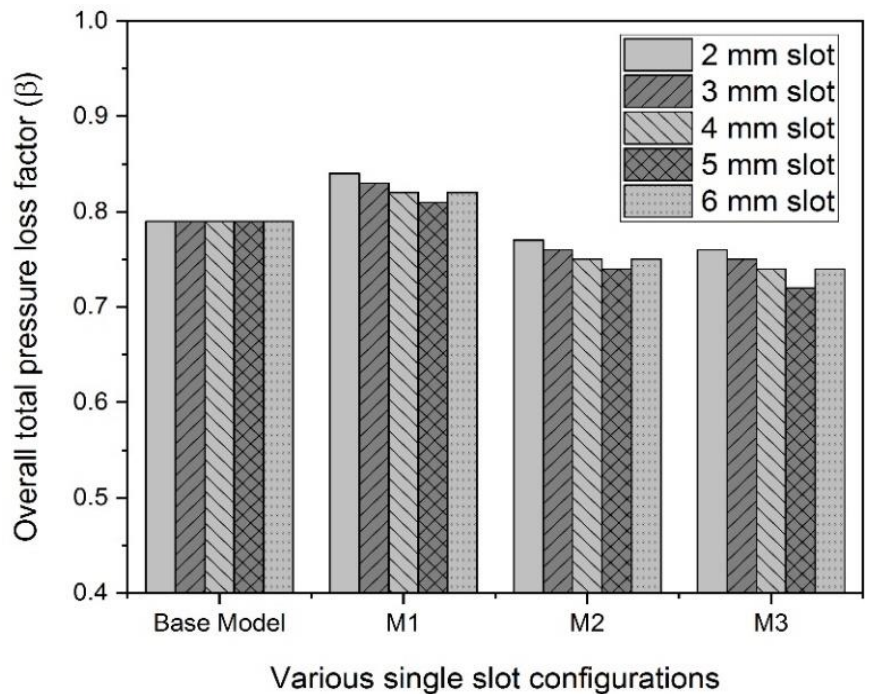

Fig. 12 Plot of overall total pressure loss factor for various single slot configurations and slot sizes.

\subsection{Effect of twin slot on the performance}

Encouraged by the result of useful improvement in performance parameters like overall static pressure rise factor and total pressure loss factor for some of the single slot configurations as explained earlier, the utility of a twin slot on the impeller blades is now being examined here. The twin slot sizes are varied from $2 \mathrm{~mm}$ to $6 \mathrm{~mm}$ with an increment of 1 $\mathrm{mm}$ in these analyses also. The effect of twin slots on flow behavior corresponding to an inlet flow velocity of $5 \mathrm{~m} / \mathrm{s}$ is taken up for discussion.

Figure 13 represents the plot of the overall static pressure rise factor for various twin slot configurations and slot dimensions. It is clear from this figure that the slotted configuration M5, corresponding to slot locations at $50 \%$ and $75 \%$ from blade root, was found to yield better results in terms of overall static pressure rise factor when compared with other twin slot configurations including the base model. However, configurations M4 and M6 corresponding to slot locations near the eye region and mid-height as well as twin slots provided at the eye region and tip region respectively are leading to adverse influence in terms of static pressure rise across the blower.

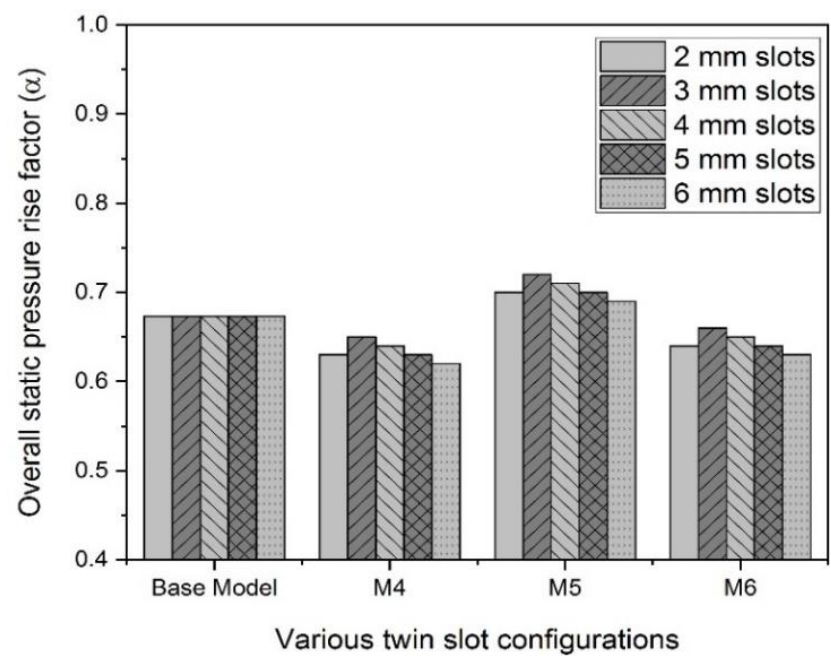

Fig. 13 Plot of overall static pressure rise factor for various twin slot configurations and slot sizes. 


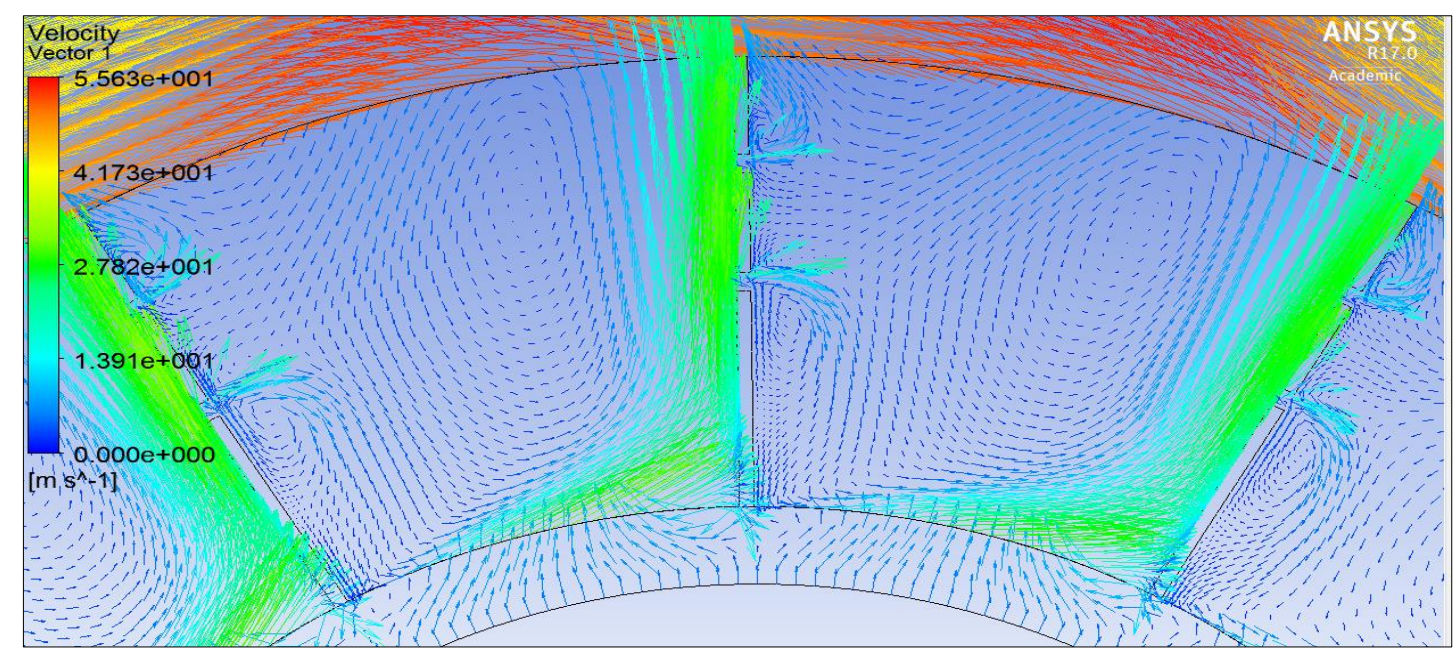

Fig. 14 Velocity vector plot of configuration M5 with optimum slot size.

The reason for the optimum performance for configuration M5 is due to the pushing of air towards volute casing more effectively by twin jets with the minimum amount of impeller tip leakage losses. This leads to a better incoming flow into the volute casing in terms of streamlining thereby contributing towards pressure recovery.

Configuration M5 having twin slots of radial height $3 \mathrm{~mm}$ is found to be superior over other slot sizes considered in the study. This slot width yields about a $7 \%$ improvement in the overall static pressure rise factor when compared with the base model configuration. This may be due to the fact that a $3 \mathrm{~mm}$ height twin jet reduces the strength of the primary vortex thereby causing an increase in pressure recovery. The velocity vector plot for the optimum configuration M5 is shown in Fig. 14. It is clearly seen that these jets create a small fluid momentum in the form of smaller local turbulent zones in the vicinity of slot locations near the suction side of the impeller. The momentum thus gained by the fluid will energize the neighboring fluid particle and this process continues until the fluid reaches the tip of the impeller. The combined triggering effect of these jets is manifested by the performance augmentation of the blower in terms of static pressure rise for this configuration.

It is seen from Fig. 13 that the performance of configuration M4 is inferior when compared with that of the base model configuration. Slots at the eye region and mid-height of the blade create pronounced recirculation zones near the jets. These zones obstruct the flow downstream thereby causing relatively higher flow losses. This results in a decremental trend for the static pressure recovery of the blower for this configuration. The velocity vector plot for this configuration in Fig. 15 clearly depicts these phenomena.

So also, configuration M6 produces a comparatively lower overall static pressure rise factor when compared with the base model configuration for all slot sizes. The reasoning for this phenomenon is explained with the help of a velocity vector plot as seen in Fig. 16. This can be ascribed to the fact that in the vicinity of the impeller eye region as well as at the impeller tip region, more pronounced disturbances are created by the suction jets. These disturbances contribute to additional

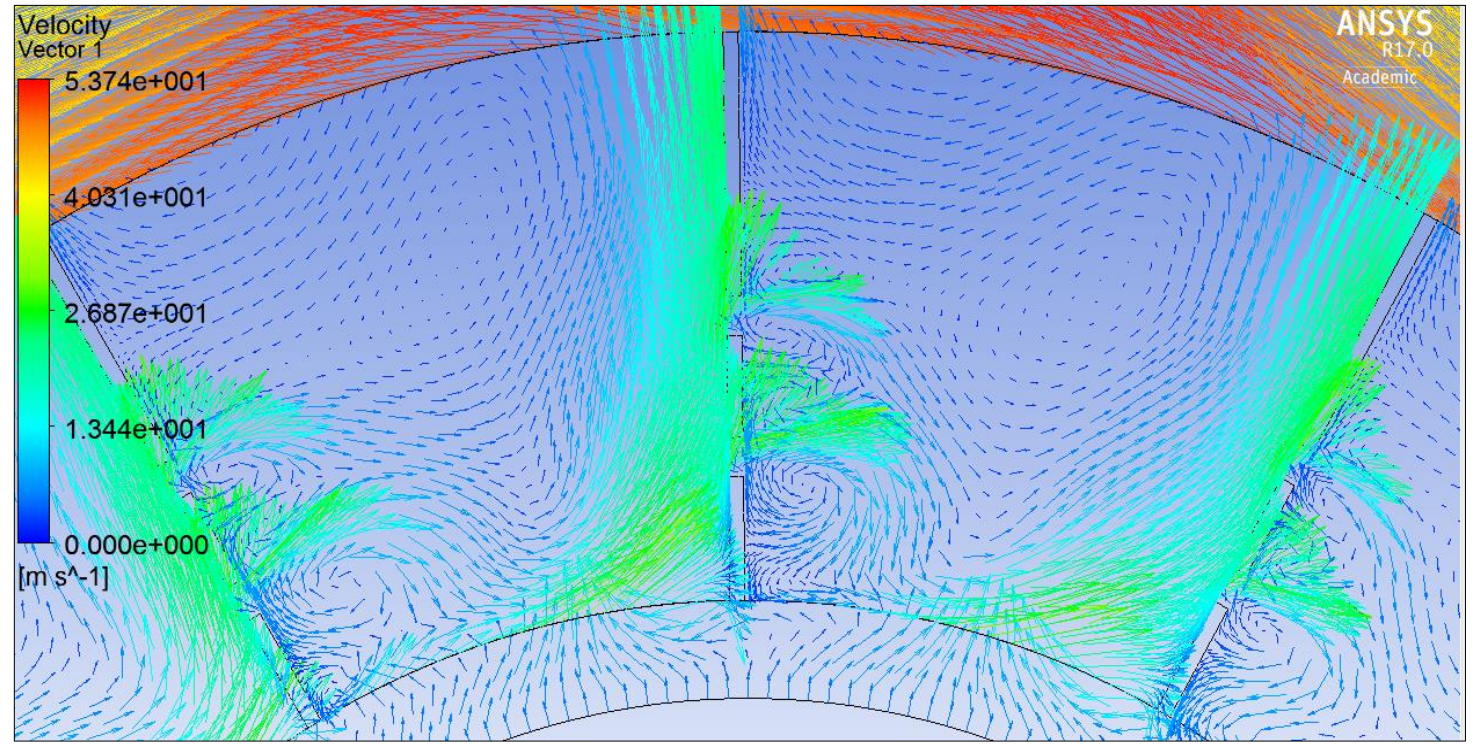

Fig. 15 Velocity vector plot of configuration M4 with optimum slot size. 


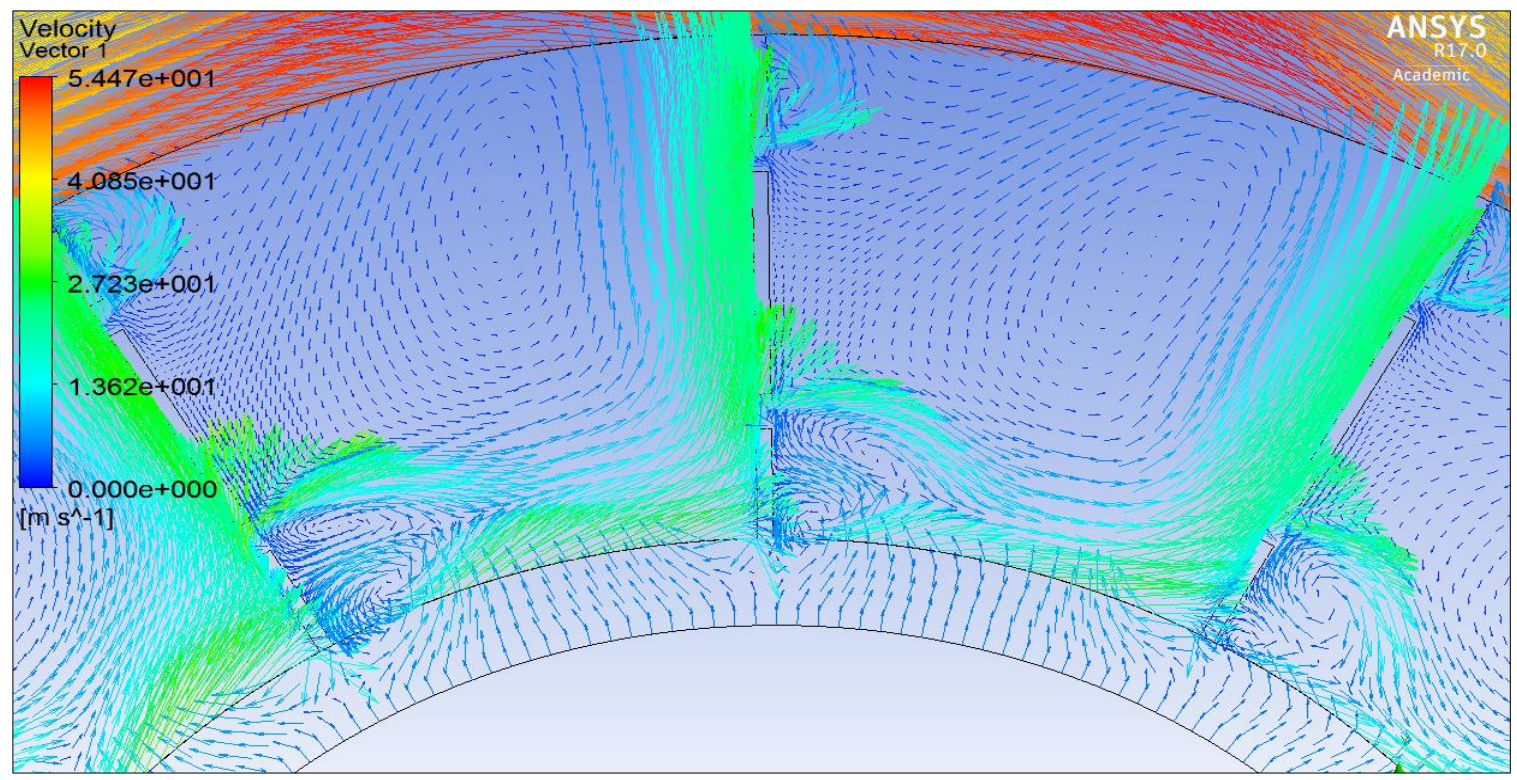

Fig. 16 Velocity vector plot of configuration M6 with optimum slot size.

turbulence for the smoother fluid flow thereby deteriorating the performance.

As a secondary check, a graph of the total pressure loss factor for all the twin slotted configurations is drawn and is displayed in Fig. 17. Configuration M5, being the optimum configuration amongst the twin slot models, yields a relatively lower total pressure loss factor across the blower when compared with other geometrical configurations. This clearly indicates that better static pressure recovery is obtained for configuration M5 thereby validating Fig. 13.

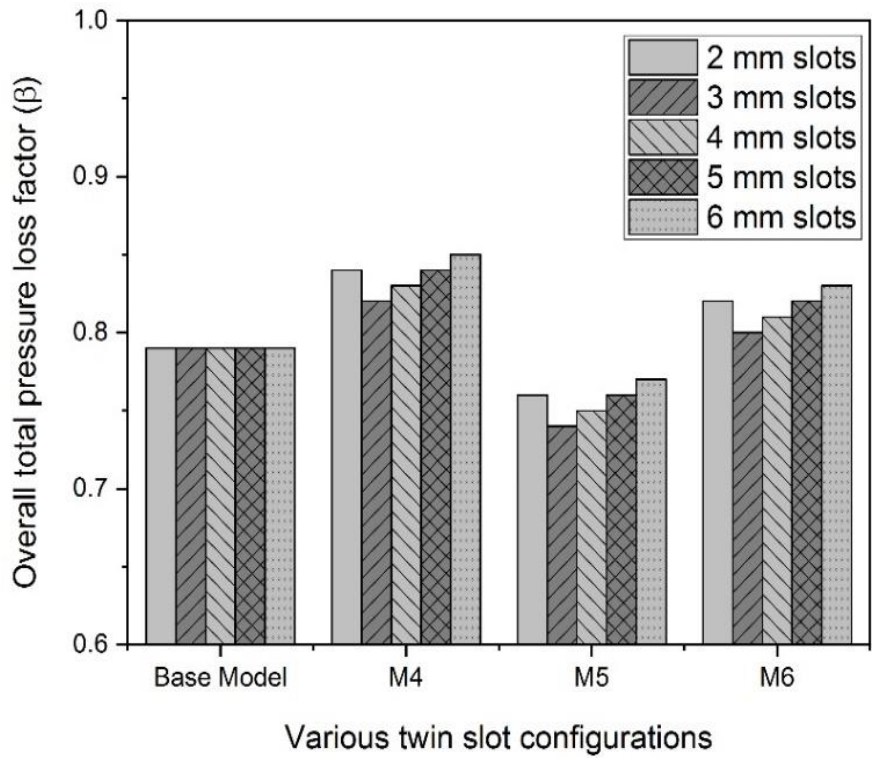

Fig. 17 Plot of overall total pressure loss factor for various twin slot configurations and slot sizes.

Finally, it can be summarized that the twin slot provided near the impeller tip as well as the mid-height of the impeller improves the overall flow characteristics of the centrifugal blower by energizing the flow stream in the impeller passages and re-aligning the flow.

\subsection{Comparative study of the effect of slot on the performance}

The results of the optimized single-slot configuration of the present analyses are compared with that of the work performed by Karanth et al. ${ }^{[6]}$ The overall static pressure rise factor is chosen as the performance parameter for the comparison purpose. Table 4. depicts the comparative analysis of two studies for the chosen performance index.

Table 4. Comparison between the present analysis and the work by Karanth et al. ${ }^{[6]}$

\begin{tabular}{|c|c|c|c|}
\hline & \multicolumn{2}{|c|}{$\begin{array}{l}\text { Overall static pressure rise } \\
\text { factor }\end{array}$} & \multirow{2}{*}{$\begin{array}{l}\text { Percentage } \\
\text { improvement }\end{array}$} \\
\hline & Base Model & $\begin{array}{l}\text { The slot at } 75 \% \\
\text { of radial height }\end{array}$ & \\
\hline Present work & 0.6800 & 0.7174 & 5.50 \\
\hline $\begin{array}{l}\text { Work by } \\
\text { Karanth } \rho+g\left[{ }^{[6]}\right.\end{array}$ & 0.2890 & 0.2940 & 1.73 \\
\hline
\end{tabular}

From Table 4, it is seen that the percentage improvement is found higher for the present study when compared with the work by Karanth et al. ${ }^{[6]}$ This indicates that the performance of the blower depends on various parameters which include geometric as well as kinematic variables.

\section{Conclusion}

From the numerical study, it is concluded that the presence of slot/s at judicious locations tends to improve the performance of centrifugal blower. The crisp outcomes of the present study are as follows:

The presence of a single slot at $75 \%$ of chord length from the blade root helps in attenuating the formation of the recirculation zone and gives a relatively higher improvement and is highly recommended to be employed. Static pressure rise increases with the slot width reaching up to an optimum value of $5 \mathrm{~mm}$ and then reduces. The optimum single slot 
configuration having optimum slot size yields about a $5.5 \%$ improvement in the overall static pressure rise factor when compared with the base model configuration. The twin slot arrangement located at a radial height of 50\% and $75 \%$ from the blade inlet surface causes prevents the formation of vortices to yield a positive effect in terms of pressure rise. So also, a $3 \mathrm{~mm}$ slot size is found to be the optimum slot size for twin slot configuration. The optimum twin slot configuration having optimum slot size yields about a $7 \%$ improvement in the overall static pressure rise factor when compared with the base model configuration.

\section{Acknowledgment}

The experimental and computational facility for conducting this research work was provided by the Department of Mechanical and Manufacturing Engineering, Manipal Institute of Technology, Manipal Academy of Higher Education (MAHE), Manipal. The authors wish to express their gratitude and acknowledge the support provided by the institute in carrying out this research work.

\section{Conflict of interest}

There are no conflicts to declare.

\section{Supporting information}

Not Applicable.

\section{References}

[1] K. Hubrich, A. Bolcs, P. Ott, Boundary layer suction via a slot in a transonic compressor: numerical parameter study and first experiments, Proceedings of the ASME Turbo Expo 2004 Power for Land, Sea and Air, Austria, 2004, 527-536, GT2004-53758, doi: 10.1115/GT2004-53758.

[2] M. H. Shojaefar, A. R. Noorpoor, A. Avanesians, M. Ghaffarpou, American Journal of Applied Sciences, 2005, 2, 1474-1480, doi: 10.3844/ajassp.2005.1474.1480.

[3] J. S. B. Gajjar, F. T. Smith, Philosophical Transactions of the Royal Society A, 2005, 363, 1199-1208, doi: 10.1098/rsta.2005.1561.

[4] Y. Song, F. Chen, J. Yang, Z. Wang, Journal of Turbomachinery, 2006, 128, 357-366, doi: 10.1115/1.2162181.

[5] D. Georgiev, S. D. Vlaev, Chemical and Biochemical Engineering Quarterly, 2008, 22, 267-272, doi: 10.15255/CABEQ.2014.345.

[6] K. Vasudeva Karanth, N. Yagnesh Sharma, Proceedings of the Institution of Mechanical Engineers, Part C: Journal of Mechanical Engineering Science, 2010, 224, 1665-1678, doi: 10.1243/09544062JMES1990.

[7] Karanth, K. Vasudeva, N. Yagnesh Sharma, World Academy of Science, Engineering and Technology, International Journal of Mechanical, Aerospace, Industrial, Mechatronic and Manufacturing Engineering, 2009, 3, 1493-1499.

[8] K. M. S. Eldalil, Proceedings of ICFD 10: Tenth International Congress of Fluid Dynamics (ICFD 10), 2010, ICFD10-EG-3017. [9] N. K. Madhwesh, V. Karanth, N. Yagnesh Sharma,
Proceedings of the World Congress on Engineering, 2011, 3, WCE 2011, London.

[10] A. V. Dovgal, A. M. Sorokin, Journal of Applied Mechanics and Technical Physics, 2006, 47, 510-514, doi: 10.1007/s10808006-0083-3.

[11] M. Gancedo, E. Guillou, E. Gutmark, International Journal of Heat and Fluid Flow, 2018, 70, 206-215, doi: 10.1016/j.ijheatfluidflow.2017.12.007.

[12] R. H. Zhang, L. C. Yun, J. Li, Journal of Hydrodynamics, 2019, 31, 733-739, doi: 10.1007/s42241-018-0161-z.

[13] H. Wang, B. Long, C. Wang, C. Han, L. Li, Energies, 2020, 13, 1628, doi: 10.3390/en13071628.

[14] N. Madhwesh, K. Vasudeva Karanth, N. Yagnesh Sharma, Journal of Mechanical Science and Technology, 2018, 32, 185 197, doi: 10.1007/s12206-017-1220-z.

[15] A. F. Ayad, H. M. Abdalla, A. Abou El-Azm Aly, Aerospace Science and Technology, 2015, 47, 247-255, doi: 10.1016/j.ast.2015.09.033.

\section{Authors information}

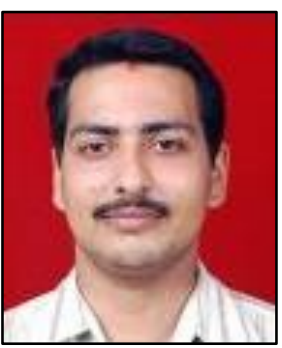

Madhwesh $\mathrm{N}$ is the Associate Professor in the Department of Mechanical and Manufacturing Engineering, Manipal Institute of Technology, Manipal Academy of Higher Education, Manipal, India. His research interests are in the areas of Turbo Machinery, Heat Transfer and Internal Combustion Engines.

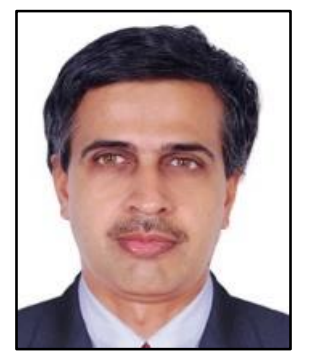

K Vasudeva Karanth presently is working as a Professor in the Mechanical and Manufacturing Engineering Department, Manipal Institute of Technology, Manipal University, Manipal, India. He obtained his Ph.D. in the field of Turbomachinery studies from Manipal Academy of Higher Education. He has expertise in the domains of Solar Energy and Turbo Machines among others. He has published many research articles in leading international journals.

Publisher's Note: Engineered Science Publisher remains neutral with regard to jurisdictional claims in published maps and institutional affiliations. 